COMMENT. These findings are confirmation of a febrile seizure threshold dependent on the height of the fever first proposed 30 years ago (Millichap JG. Studies in febrile seizures I. Height of body temperature as a measure of the febrile seizure threshold. Pediatrics $1959 ; 23: 76)$. It is not surprising that children who convulse with a relatively low fever have a poorer prognosis than those convulsing only with high fevers. Another factor noted in many studies to increase the risk of recurrence of febrile seizures is an early age at the first febrile seizure. Frantzen et al (1968) found that children who experienced their first seizure at less than 13 months of age had a 2.3:1 chance for developing further febrile seizures compared to a $1: 2$ chance when the seizure occurred between 14 and 32 months of age and a 1:5 risk when the onset was after 32 months of age. Nelson and Ellenberg (1978) found a $50 \%$ recurrence rate with the onset in the first year of life campared to a $28 \%$ recurrence rate when the onset was after the first year. The height of the fever is a most important determinant of the occurrence of febrile seizures in the infant and young child.

\title{
NEURODEGENERATIVE DISORDERS
}

\section{NEUROAXONAL DYSTROPHY}

The clinical, pathological and biochemical findings in two brothers with a newly recognized form of infantile neuroaxonal dystrophy associated with alpha-N-acetylgalactosaminidase deficiency are reported from the Divisions of Medical and Molecular Genetics and Neuropathology, Mount Sinai School of Medicine, New York; Department of Chemistry, University of Alberta, Edmonton, Canada; Department of Physiological Chemistry, University of Bonn, Federal Republic of Germany; and Department of Hum Genetics, University of Wurzburg, Federal Republic of Germany. The brothers were the offspring of fifth cousins of German descent and their early development was normal. In the older brother, the clinical onset of disease was signaled by poor coordination of gait, clumsiness, and episodes of falling at 12 months. In the younger brother, grand mal seizures began at eight months and occurred five times over the next six months. Each had a regressive course beginning after 15 months of age, with loss of all mental and motor skills acquired previously. Strabismus, nystagmus, vi sual impairment, muscular hypotonia, and frequent myoclonic movements developed in both. By three to four years of age, both brothers had profound psychomotor retardation and spasticity, and were immobile and incontinent. CT and MRI showed atrophy of the cerebellum, brain stem and cervical spinal cord, with lesser atrophic changes in the cerebral gray and white matter, optic tracts and cranial nerves. EFG revealed multifocal isolated spikes and spike wave complexes. The urinary oligosaccharide profile was abnormal, suggesting the possibility of a lysosomal disease. The activity of alpha-N-acetylgalactosaminidase was deficient whereas 21 other lysosamal enzymes were normal. (Schindler D et al. Neuroaxonal dystrophy due to lysosamal alpha-N-acetylgalactosaminidase deficiency. N Engl J Med June 29,$1989 ; 320: 1735-1740$ ). 
COMMENT. The neuroaxonal dystrophies include the infantile (Seitelberger's disease, late-infantile, and juvenile forms, neuroaxonal leukodystrophy, and Hallervorden-Spatz symdrome) and all are characterized by the spheroids in the terminal endings of axons in the central nervous system. The axonal dystrophy in alpha-Nacetylgalactosaminidase deficiency described in this article differs from that observed in other lysosamal storage diseases. The absence of identifiable lysosomal storage in this newly described disorder suggests a causal relation between the enzyme deficiency and the resultant axonal pathology. A report from the Departments of Pediatrics, Radiology and Neurology, Kyoto, Japan, suggests that magnetic resonance imaging may be useful in the diagnosis and classification of infantile neuroaxonal dystrophy ( $I$ to $M$ et al. Pediatr Neurol 1989; 5:245-8). A six year old boy with typical clinical features of infantile neuroaxonal dystrophy showed increased metal deposition in the globus pallidus and MRI findings of Hallervorden-Spatz syndrame suggesting that these two disease entities overlap. The "eye of the tiger" sign was described in the MRI of two patients with Hallervorden-Spatz syndrome and dystonia. (See Ped Neur Briefs, October 1988, 2:77).

RETT SYNDROME

The clinical peculiarities and differential diagnosis of Rett syndrame are reviewed from the Department of Pediatrics Children's Clinics, East Hospital, Goteborg, Sweden. The four clinical stages of classic Rett syndrame are as follows. 1) Early onset stagnation, 2) rapid developmental regression, 3) pseudostationary period, 4) late motor deterioration. A variety of atypical variants have been described including some in boys. The development during the first few months of life is sometimes abnormal, rarely there is no subsequent deterioration phase, and occasionally seizures occur early as infantile spasms. The term "formes frustes" has been coined for these abortive variants. The differential diagnosis in Rett syndrame stage 2, which includes rapid developmental regression, increased irritability, screaming episodes and loss of acquired skills, is as follows: Infantile neuronal ceroid lipofuscinosis, encephalitis, toxic encephalopathies, epileptic encephalopathies, infantile autism, neurocutaneous syndromes, glutaric aciduria, amino acidopathy, and ataxic cerebral palsy. Biologic markers and effective screening procecures for an early diagnosis are lacking. A possible viral origin with a disorder similar to SSPE is now being suggested as an etiology of this syndrome. (Hagberg BA. Rett syndrome: Clinical peculiarities, diagnostic approach, and possible cause. Pediatr Neurol Mar/Apr 1989; 5:75-83).

COMMENT. As the author concludes, the Rett syndrome concept is broade $\bar{r}$ than previously believed and atypical variants must be recognized. Autopsy findings have been surprisingly limited even in advanced stages of the disease and have included moderate cortical atrophy and general brain shrinking with increasing age. Microscopically, there was mild gliosis without evidence of storage, underpigmentation in certain nigral structures, and axonal changes suggestive of degeneration in ascending and descending tracts. 